Subscriber access provided by Caltech Library

\title{
Article
}

\section{Broadband sensitization of lanthanide emission with indium phosphide quantum dots for visible to NIR downshifting} Joseph K. Swabeck, Stefan Fischer, Noah D Bronstein, and A. Paul Alivisatos

J. Am. Chem. Soc., Just Accepted Manuscript • DOI: 10.1021/jacs.8b02612 • Publication Date (Web): 20 Jun 2018

\section{Just Accepted}

"Just Accepted" manuscripts have been peer-reviewed and accepted for publication. They are posted online prior to technical editing, formatting for publication and author proofing. The American Chemical Society provides "Just Accepted" as a service to the research community to expedite the dissemination of scientific material as soon as possible after acceptance. "Just Accepted" manuscripts appear in full in PDF format accompanied by an HTML abstract. "Just Accepted" manuscripts have been fully peer reviewed, but should not be considered the official version of record. They are citable by the Digital Object Identifier (DOI®). "Just Accepted" is an optional service offered to authors. Therefore, the "Just Accepted" Web site may not include all articles that will be published in the journal. After a manuscript is technically edited and formatted, it will be removed from the "Just Accepted" Web site and published as an ASAP article. Note that technical editing may introduce minor changes to the manuscript text and/or graphics which could affect content, and all legal disclaimers and ethical guidelines that apply to the journal pertain. ACS cannot be held responsible for errors or consequences arising from the use of information contained in these "Just Accepted" manuscripts. 


\title{
Broadband sensitization of lanthanide emission with indium phos- phide quantum dots for visible to NIR downshifting
}

\author{
Joseph K. Swabeck ${ }^{1,2}$, Stefan Fischer ${ }^{1,2}$, Noah D. Bronstein ${ }^{1,2}$, and A. Paul Alivisatos, $1,2,3,4,{ }^{*}$ \\ ${ }^{1}$ Materials Sciences Division, Lawrence Berkeley National Laboratory, Berkeley, California 94720, USA \\ ${ }^{2}$ Department of Chemistry, University of California-Berkeley, Berkeley, California 94720, USA \\ ${ }^{3}$ Department of Materials Science and Engineering, University of California-Berkeley, Berkeley, California 94720, \\ USA \\ ${ }^{4}$ Kavli Energy NanoScience Institute, Berkeley, California 94720, USA
}

\begin{abstract}
Semiconductor quantum dot sensitized lanthanide ions hold great promise in producing a broadly absorbing and sharply emitting luminophore, but their synthesis has proven to be difficult. We report the first synthesis of core/shell/shell $\mathrm{InP} / \mathrm{Ln}_{\mathrm{x}} \mathrm{Y}_{1-\mathrm{x}} \mathrm{F}_{3} / \mathrm{ShF}_{3}(\mathrm{Ln}=\mathrm{Yb}, \mathrm{Nd}, \mathrm{Sh}=\mathrm{Lu}, \mathrm{Y})$ nanocrystals that exhibit a broad visible absorption coupled to a sharp nearinfrared emission. Additionally, this is the first report of $\mathrm{Nd}$ being coupled to a quantum dot absorber. We characterize the system with a variety of electron microscopy and x-ray techniques that prove this unique structure. Optical measurements confirm the correlation of the $\mathrm{Ln}^{3+}$ emission to the quantum dot ab-sorption, while the presence of a trap state emission gives a clue as to the mechanism of energy transfer between the dot and the lanthanide.
\end{abstract}

\section{Introduction}

Trivalent lanthanide ions (Ln) are widely used as emission centers in various optical applications, thanks to their manifold of sharp emissions that span the visible and near infra-red (NIR) spectrum. This has led to their use in energy conversion schemes ${ }^{1-3}$, bioimaging 4,5 , and other applications where a sharp absorption or emission line is desira$b^{6}{ }^{6}$. Ln ions also exhibit long luminescence lifetimes, allowing for photophysical interactions such as up and down conversion ${ }^{7}$. Along with these benefits come a variety of challenges. The fact that the transitions within these ions are atomic-like means that the range of energies that can be absorbed by these ions is very small, limiting their potential applications ${ }^{8}$. For example, the narrow absorption of these materials would make them unsuitable for solar energy conversion. Additionally, the forbidden nature of the f-f transitions mean that the absorption cross section is very small compared to dyes or quantum dots, necessitating large numbers of ions in order to have practical absorption values within a luminophore, which can result in an increase of lossy processes, such as cross relaxation and energy migration to non-radiative quenching centers.

A variety of methods have been employed to try to overcome one of this limiting factor of Ln ions, the weak absorption, while maintaining their narrow linewidths. These methods include the use of organic molecules, other Ln ions with higher absorption cross section, and quantum dots (QD's). Organic dyes and complexes ${ }^{9}$ have long been employed as sensitizing agents for Ln ion transitions, with photoluminescent quantum yields (PLQYs)of 75\% being reported. ${ }^{10}$ Though the absorption of the Ln ions is improved with these materials, they still do not offer a broad band absorption profile or the level of control that is necessary for achieving very high (>95\%) PLQYs. Due to the lack of a protective shell in the materials, nonradiative quenching processes are present and can have a large impact on the PLQY of the material. ${ }^{11}$ Other Ln ions, such as cerium, have been used as high energy absorbers coupled to emissive Ln species. These systems use the high energy $4 f-5 d$ transitions that, in contrast with the $4 f-4 f$ transitions that are observed in Ln ions, are more allowed. Though these are not as strong as $s-p$ transitions, they are still much stronger than the $f-f$ transitions that are normally observed. This significantly increases the cross section of the absorption, and can result in more efficient downshifting and downconversion ${ }^{12}$ processes. This method of sensitization is limited to high energy ( $>4.5 \mathrm{eV}$ in fluoride hosts ${ }^{13}$ ) photons, which limits its usefulness in most applications.

QD's have a number of desirable properties as potential sensitizers for Ln ions. Since QD's are semiconductors, they have a strong broadband absorption above their band gap. The band gap of a QD is tunable via its size, shape, composition, and shell material. ${ }^{14,15}$ This allows for the selection of the absorption profile that is best suited for the application at hand. Additionally, the tunability of the band gap allows for changing the energy offset between the QD and the Ln ion, which allows engineering of the transfer rate. Previous 
A
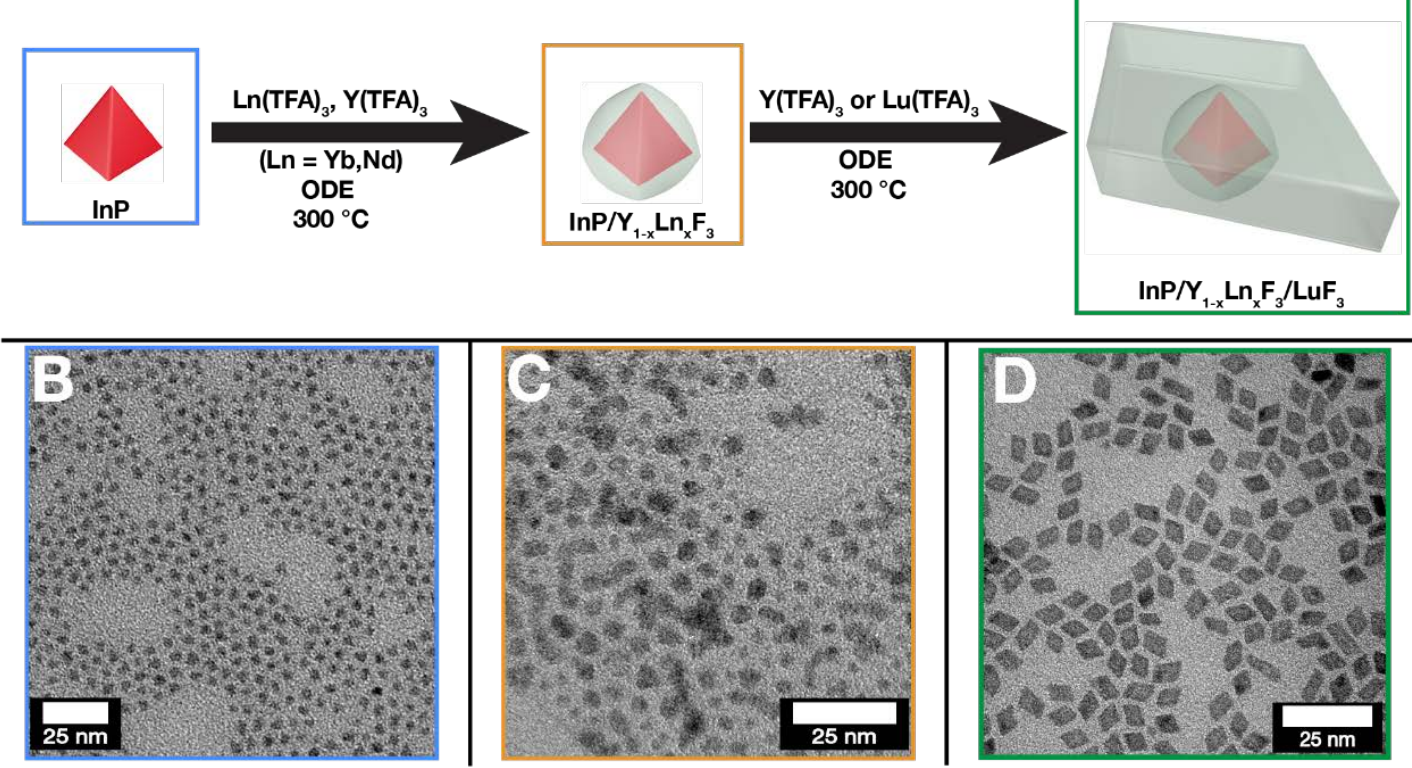

Figure 1. Overview of the synthesis of core/shell/shell nanocrystals. (a) Schematic representation of the overall synthesis of the core/shell/shell nanocrystals. (b) TEM image of the core InP QDs before any shelling or surface treatments. (c) TEM image of InP/ $\mathrm{Y}_{1-\mathrm{x}} \mathrm{Yb}_{\mathrm{x}} \mathrm{F}_{3}$ core/shell nanocrystals. (d) TEM image of the completed InP/ $\mathrm{Y}_{1-\mathrm{x}} \mathrm{Yb}_{\mathrm{x}} \mathrm{F}_{3} / \mathrm{LuF}_{3}$ core/shell/shell nanocrystals after size selective precipitation was used to create a monodisperse sample

work by the Meijerink group showed that it was possible to combine the absorption of a QD with the emission of a lanthanide ion ${ }^{16}$. The adsorption of $\mathrm{Yb}^{3+}$ on the surface of cadmium selenide (CdSe) QDs showed energy transfer from the $\mathrm{QD}$ to the $\mathrm{Yb}^{3+}{ }^{2} \mathrm{~F}_{5 / 2}$ state, creating an emission in the NIR. This work also showed that surface passivation of this kind of system was key, though even with an overgrowth of a selenium shell around the adsorbed $\mathrm{Yb}^{3+}$, the emission from the Ln was fairly weak and no PLQY values were reported. Other recent work by the Gamelin group showed that stable particles could be made via a cation exchange. ${ }^{17}$ This work reports PLQY 5\%, though improvements may be difficult, as a large passivation layer is necessary for optimum PLQY, and shell growth on $\mathrm{PbIn}_{2} \mathrm{~S}_{4}$ has not been deeply explored. Additional recent work by the Gamelin group has shown the use of $\mathrm{CsPbCl}_{3}$ as a host for $\mathrm{Yb}^{3+}$ ions that, when excited at high energies, results in a quantum cutting effect that can produce PLQY values of over $170 \% .18$ These particles have impressive luminescence properties, but the narrowness of the absorption, stability of the perovskite host, and concerns about the toxicity of lead will hinder their widespread use.

This work investigates a stable downshifting configuration that combines the broad absorption of an indium phosphide (InP) QD with the narrow emission of the $\mathrm{Yb}^{3+}$ ion. This is done by coating the InP QD with a shell of ytterbiumdoped yttrium trifluoride $\left(\mathrm{Y}_{1-\mathrm{x}} \mathrm{Yb}_{\mathrm{x}} \mathrm{F}_{3}\right)$, and growing a final passivating shell of $\mathrm{LnF}_{3}$, where $\mathrm{Ln}$ is $\mathrm{Y}$ or $\mathrm{Lu}$. This core/shell/shell structure was found to undergo energy transfer when exciting the InP above its band edge to the $\mathrm{Yb}^{3+}{ }^{2} \mathrm{~F}_{5 / 2}$ state, where a sharp emission at $976 \mathrm{~nm}$ was observed.

\section{Results and Discussion}

An overview of synthesis of the $\mathrm{InP} / \mathrm{Yb}_{\mathrm{x}} \mathrm{Y}_{1-\mathrm{x}} \mathrm{F}_{3} / \mathrm{LuF}_{3}$ core/shell/shell nanocrystals is shown in Figure $1 \mathrm{a}$. Thought this scheme has been extened to $\mathrm{Nd}$, the majority of this paper will focus on $\mathrm{Yb}$ as the luminescent $\mathrm{Ln}$ ion due to its simplicity. Full synthetic procedures can be found in the Supplemental Information. Briefly InP cores were synthesized according to literature procedures with minor changes ${ }^{19}$. It is very important to note that trace oxygen makes a large difference in the properties of the InP nanocrystals that are formed. Even using 99.9995\% Ar still results in a significant amount of oxygen on the surface of the particle, and this oxygen shell improves the luminescence efficiency of the un-shelled nanocrystal by orders of magnitude, reaching around 3\% efficiency for band-edge emission for heavily oxidized particles. Additionally, it was found that in an earlier version of this procedure, where $\mathrm{Yb}$ was introduced into the synthesis of InP, that moving from standard purity argon (99.5 \%) to ultra-high purity argon (99.9995\%) resulted in a marked decrease in the NIR luminescence from the $\mathrm{Yb}^{3+}$, as well as no evidence for $\mathrm{Yb}-\mathrm{P}$ bonds through extended $\mathrm{x}$-ray absorption fine structure (EXAFS). Further purifying the argon to sub part-per-billion oxygen resulted in no NIR luminescence. We believe that this is because removing the oxide species from the surface results in an In rich facet that would not favor $\mathrm{Yb}$ binding. This suggests that the reactive oxygen content of a standard Schlenk line results in an oxide covered InP surface, and that the surface oxide is necessary for the adsorption of $\mathrm{Yb}$ to InP. A more thorough treatment of these effects can be found in the SI. The inablity of $\mathrm{Yb}$ to adhere to non-oxidized crystals resulted in the current scheme of using the fluorides as the lanthanide host wrather than relying on surface 

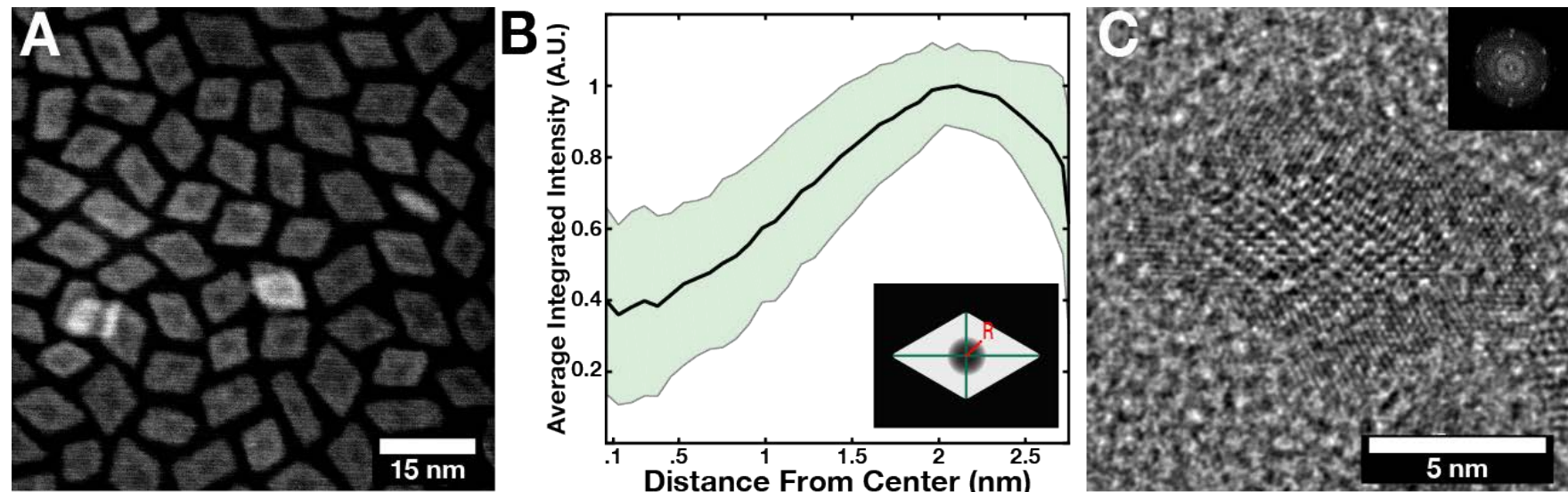

Figure 2. Structural characterization via electron microscopy. (a) HAADF-STEM image of the core/shell/shell nanocrystals The contrast difference that is observed is due to the $\mathrm{Z}^{2}$ contrast function of HAADF-STEM, where lighter elements appear darker in the image. (b) Radially integrated contrast values of 86 particles and the standard deviation of that measurement, with lower contrast giving lower integrated intensity. The inset shows a cartoon of the process that was done to make this graph, and a more detailed treatment can be found in the SI. (c) HRTEM image of a core/shell/shell nanocrystal showing the lattice spacings of both the core and the shell materials of the nanocrystal. The inset FFT also shows the clear signature of two independent lattices being present in the image.

adsorption. Due to the insensitivity of the lanthanide fluorides to the surface oxidation of the InP, they have proven to be a more robust method of adhering lanthanides to QD's.

Cleaned InP nanocrystals were next treated with tetrabutylammonium fluoride (TBAF) to passivate the surface, remove any trace oxygen that was on the surface of the particle by replacing it with fluoride, and provide a fluoride rich layer for the rare earth ions to adhere to, and later shelled using yttrium trifluoroacetate $\left(\mathrm{Y}(\mathrm{TFA})_{3}\right)$ and $\mathrm{Yb}(\mathrm{TFA})_{3}$ to create the optically active layer. The treatment of InP with fluoride ions has been investigated previously, and our work uses similar methods to the literature. ${ }^{20,21}$ Finally, an inert shell was grown using $\mathrm{Y}(\mathrm{TFA})_{3}$ or Lu(TFA $)_{3}$ to complete the synthesis. An overview of this synthesis, along with the corresponding TEM images at each step, is shown in Figure 1. It should be noted that the parallelogram

morphology of the final particles is consistent with the previous reports of $\mathrm{LuF}_{3}$ nanoplatelets in the literature. ${ }^{22}$ Additionally, it should be noted that a size slective precipitation was used to remove small impurities and achieve the final product. If the reaction mixture is used, there is some degree of inhomogeneity in the particles (SI), though the size selected particles display a high degree of homogeneity.

Further electron microscopy characterization was performed to confirm the core/shell/shell morphology of the product nanocrystals. Figure 2a shows a HAADF-STEM image of the particles shown in Figure 1d. Since HAADF-STEM has atomic number contrast, one would expect the center of the nanocrystal to appear darker, corresponding to the lighter indium and yttrium that should be present there as compared to the lutetium that makes up the outermost shell.

This contrast difference can be quantified by starting at the center of each particle and radially integrating the contrast as a function of distance from the center. Figure $2 b$ shows the result of doing this integration over 86 different particles and averaging the resultant profiles for the first 2
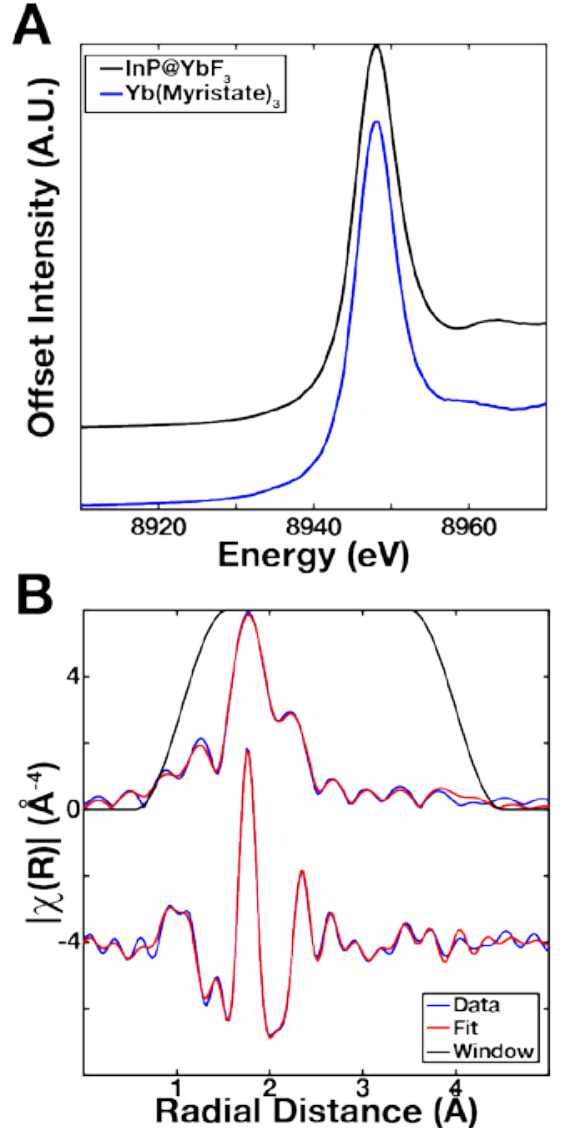

Figure 3. Yb LIII edge EXAFS. (a) XANES comparison of the $\mathrm{Yb}$ found in the core/shell nanocrystals to the $\mathrm{Yb}$ (Myristate) 3 reference with a white line value of $8947 \mathrm{eV}$. Note the differences in the EXAFS region between the two materials. (b) EXAFS data and fit of the core/shell/shell nanocrystals. The fit shown is to $\mathrm{YbF}_{3}$.

$\mathrm{nm}$, with the inset showing a schematic of the process. This gives a quantification of the contrast difference, with a lower contrast value corresponding to a lower atomic number, and that difference is in line with what would be expected from an In and Y core. In order to quantify the 
contrast in the STEM images and the formation of a coreshell nanoparticle, we calculated the expected contrast for the particles shown in Figure 2b. This was done by assuming a particle height of $\sim 4 \mathrm{~nm}$, which is observed from particles on their side in the TEM (see SI), and an InP/ $\mathrm{Y}_{1-\mathrm{x}} \mathrm{Yb}_{\mathrm{x}} \mathrm{F}_{3}$ core size of $\sim 3.7 \mathrm{~nm}$. Next, we converted these values into the number of unit cells of InP and $\mathrm{Y}_{1-\mathrm{x}} \mathrm{Yb}_{\mathrm{x}} \mathrm{F}_{3}$ multiplied them by the atomic number squared of In and $\mathrm{Y} / \mathrm{Yb}$. This number was then compared to a full stack of Lu atoms giving an expected contrast of 0.43 , which is in agreement with the observed contrast difference shown in Figure $2 b$. As one moves away from the center of the particle, the $\mathrm{LuF}_{3}$ shell becomes more dominant, and the contrast goes to a normalized value of 1 beyond the core of the nanocrystal. Since the nanocrystals have a short axis, the integrated contrast decreases once the length becomes greater than the short axis of the particle.

Further confirmation of the core-shell geometry of the particles is found in the high resolution TEM image (HRTEM) shown in Figure 2c. In this image, two different lattices are seen to be overlapping in one particle. A Fourier filtering analysis (see SI) reveals that the two lattices correspond to the [110] of InP at the core of the nanocrystal and the [001] of $\mathrm{LuF}_{3}$ in the shell. The alignment of both the core and shell along the imaging axis of the TEM was a rare event, but for the three nanocrystals that were observed with lattice fringes for both the core and the shell in HRTEM have the FFT spots of the InP [110] offset from those of the $\mathrm{LuF}_{3}[001]$ by $30^{\circ}$. The $\mathrm{LuF}_{3}$ lattice spacing in the shell $(0.31$ $\mathrm{nm}$ ) is expected from previous reports of these orthorhombic $\mathrm{LuF}_{3}$ shifted blue and broadened from its initial parallelograms. ${ }^{22}$ The lattice spacings of the $\mathrm{Y}_{1-\mathrm{x}} \mathrm{Yb}_{\mathrm{x}} \mathrm{F}_{3}$ shell were not observed due to their similarity to the [001] plane of $\mathrm{LuF}_{3}$.

With the geometry of the core/shell/shell nanoparticles confirmed, we then turn to look at the local environment of the $\mathrm{Yb}^{3+}$ dopants. In early versions of this synthesis, it was found that the $\mathrm{Yb}$ ions would adhere to surface oxides on the InP nanocrystals. Because of this, it was necessary to investigate the local structure around the $\mathrm{Yb}$ to make sure that an oxide species was not being formed. In order to do this, we used x-ray absorption near edge spectroscopy/extended x-ray absorption fine structure (XANES/EXAFS) to see if the $\mathrm{Yb}^{3+}$ dopants are in the expected fluoride matrix. Looking at the $\mathrm{Yb}^{3+}$ XANES in the core/shell/shell nanocrystals (Figure 3a), one can see that it is in the expected $3+$ oxidation state with a white line absorption energy value of $8947 \mathrm{eV}$. This is unsurprising, as that is the oxidation state that was added to the reaction mixture and no strong reducing agents were added, but even in the unprocessed EXAFS region one can see the difference in the intensities between the $\mathrm{Yb}^{3+}$ in the core/shell/shell nanocrystals and the $\mathrm{Yb}$ (myristate) 3 standard that was used. Extracting the radial distribution function of the EXAFS gives the curves shown in Figure $3 \mathrm{~b}$, which are found to be in good agreement with the $\mathrm{YbF}_{3}$ crystal structure (PDF number 01-071-1161). This agreement is far better than for any other $\mathrm{Yb}$ crystal structure we used, such as $\mathrm{Yb}_{2} \mathrm{O}_{3}$ or $\mathrm{YbOF}$ (see SI). This confirms that the $\mathrm{Yb}$ dopants in the system are in the desired matrix within the core/shell/shell particles.
Next, we studied the optical properties of the core/shell/shell system. Figure 4 a shows the absorption and photoluminescence (PL) spectra that are obtained from the core/shell/shell nanoparticles. The absorption is very indicative of the InP core nanocrystals, showing that the initial particles have slightly etched and broadened in size distribution during the shell growth process, as the absorption peak has shifted blue and broadened from its initial position (see SI). Additionally, the size of the core is in good agreement with the size that would be expected for the InP core based on both the TEM shown in Figure 1c as well as the size given by the location of the first exciton in the absorption spectrum. A calculation ${ }^{15}$ of the size based on the first exciton of the InP shows that it should be $3.5 \mathrm{~nm}$, in agreement with the $\sim 3.5 \mathrm{~nm}$ seen in the STEM contrast in Figure $2 b$.

The PL spectra of the core/shell/shell nanocrystals show three distinct peaks that correspond to different emissive states within the crystals. The first peak centered at $549 \mathrm{~nm}$ is attributed to the InP band edge emission (consistent with other works using InP of this size $)^{19}$, which shows that the energy transfer to the $\mathrm{Yb}$ ions is not complete. The broad peak centered at $728 \mathrm{~nm}$ corresponds to an interband trap state that is between the band edge of the InP and the $\mathrm{Yb}^{3+}$ ${ }^{2} \mathrm{~F}_{5 / 2}$ state, a level that has been proposed in $\mathrm{Yb}^{3+}$ implanted InP thin films. ${ }^{23}$ Finally, the sharp peak centered at $976 \mathrm{~nm}$ corresponds to the $\mathrm{Yb}^{3+}{ }^{2} \mathrm{~F}_{5 / 2}$ to ${ }^{2} \mathrm{~F}_{7 / 2}$ transition. The inset shows an expanded view of this peak at higher illumination intensity, where the increased incident flux results in greater definition of the peak shape. The characteristic peak shape of $\mathrm{Yb}^{3+}$ clearly identifies this peak, with the sharp feature at $976 \mathrm{~nm}$ being accompanied by a smaller, broader feature peaking at slightly longer wavelength. Using an integrating sphere, we measured a PLQY of $0.1 \%$ to $0.5 \%$ depending on the sample for excitation into the $\operatorname{InP}\left(\lambda_{\mathrm{ex}}=440\right.$ $\mathrm{nm}$ ) and emission from the $\mathrm{Yb}$ feature at $976 \mathrm{~nm}$. The differing PLQY comes from differences in the syntheses of this material, and we found that different fluoride treatment conditions had a large effect on the final PLQY of the system. We have seen that InP that has a higher PLQY after the fluoride treatment step tends to have a higher NIR PLQY from the $\mathrm{Yb}$ when the core/shell/shell materials are made. We believe this is due to a more complete surface fluoridation, or possibly the more complete removal of oxygen from the InP surface. Integrating the full spectrum, we observe a PLQY of 3.5\% for emission from the InP band edge, trap, and Yb combined.

The importance of the interband trap state seen in the PL is confirmed when looking at the excitation scan of the core/shell/shell nanocrystals (Figure $4 \mathrm{~b}$ ). The absorptance of InP tracks very well with the excitation scan of the 976 $\mathrm{nm}$ peak above the band gap of the InP, but below the band edge there is a peak in the excitation scan that is not explained by the QD absorption but agrees well with the trap state mentioned above. This result gives insight into the path of energy transfer from the InP to the $\mathrm{Yb}$, as this is evidence of an intermediate state that has thus far not been observed. Since the PLE scan shows absorption events that lead to an emission, the presence of this sub-gap state shows that there is a state at that energy that can be excited to produce an $\mathrm{Yb}$ luminescence. This state is not strongly 

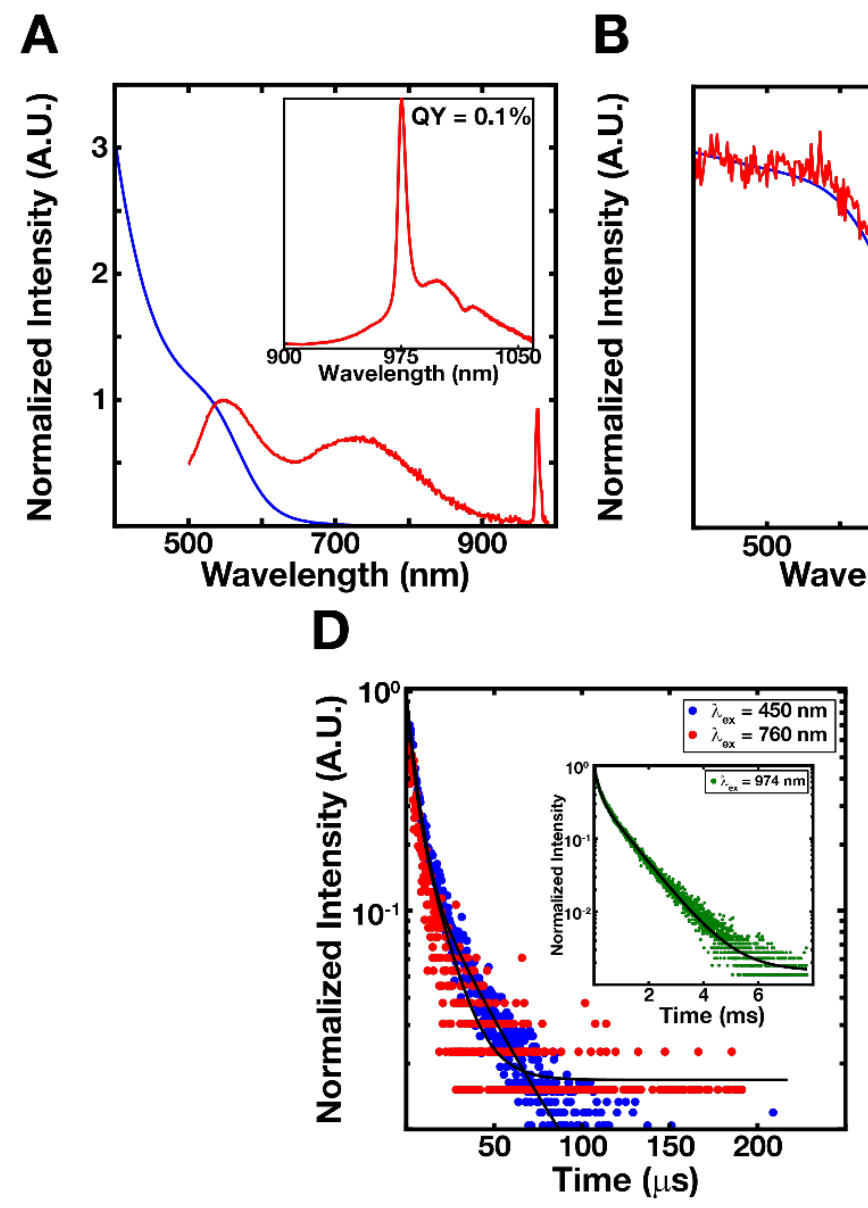

C

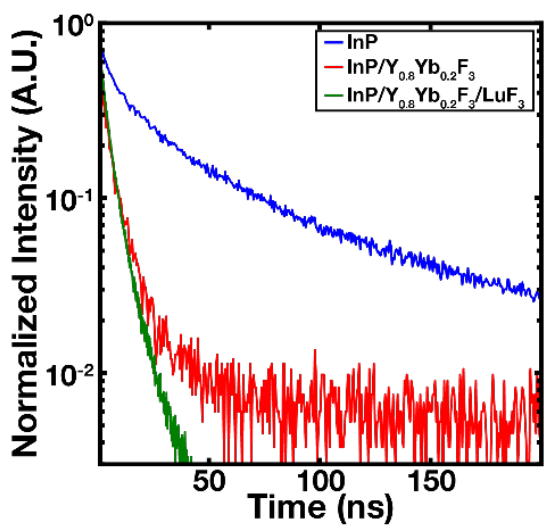

E

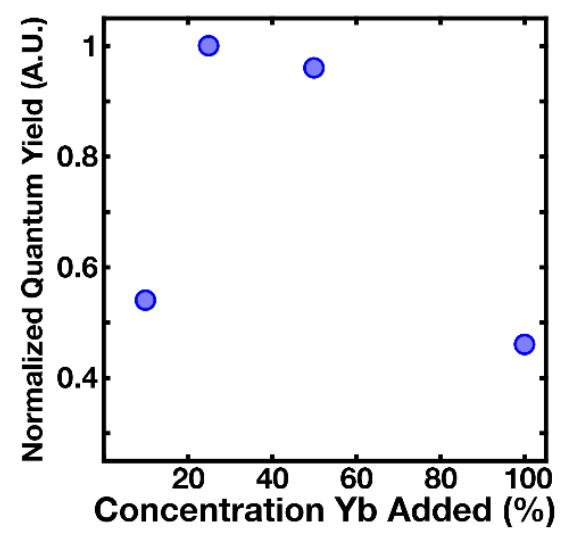

Figure 4. Optical Data. (a) Absorption (blue) and PL $\left(\lambda_{\text {ex }}=440 \mathrm{~nm}\right)(\mathrm{red})$ of the core/shell/shell nanocrystals. The inset shows the $\mathrm{Yb}^{3+}$ emission centered at $976 \mathrm{~nm}$, again under $440 \mathrm{~nm}$ excitation. (b) Absorptance (blue) vs PLE of the $976 \mathrm{~nm}$ emission (red). Note the increase in PLE after $700 \mathrm{~nm}$. (c) InP band edge emission ( $\lambda_{\mathrm{em}}=520 \mathrm{~nm}$ ) of native QDs (blue), core/shell nanocrystals (red), and core/shell/shell nanocrystals (green). A zoomed in plot of the first $200 \mathrm{~ns}$ is found in the SI. (d) Lifetimes of the $\mathrm{Yb}^{3+}{ }^{2} \mathrm{~F}_{5 / 2}$ to ${ }^{2} \mathrm{~F}_{7 / 2}$ NIR emission under different excitation wavelengths: $450 \mathrm{~nm}$ (blue), $760 \mathrm{~nm}$ (red), and $974 \mathrm{~nm}$ (green, inset). (e) Normalized PLQY under $440 \mathrm{~nm}$ excitation for various $\mathrm{Yb}$ concentrations.

absorbing (see full spectrum absorption measurements in the SI), which accounts for the fact that it does not appear in the absorptance measurements. The fact that it is observed as a positive feature in the PLE spectrum above the absorption spectrum indicates that exciting the trap directly results in more efficient PL out of the Yb ions, most likely due to increased efficiency of energy transfer to those ions. The relatively narrow breadth of the peak could be due to the shallow nature of the Ln induced trap states that lead to an emission event.

Further mechanistic insight can be derived from the time resolved PL spectra in Figure 4c and 4d. The shorter band edge lifetimes of the core/shell and core/shell/shell nanoparticles suggest that some additional processes are occurring that result in the depopulation of the band edge. The identity of these processes is not obvious, whether it is due to energy transfer processes, other nonradiative processes, or a combination of the two. What can be seen is that the band edge lifetime is shortened to similar values in both the core/shell and core/shell/shell geometries. This would suggest that the improved luminescence seen in the core/shell/shell nanoparticles is due to in increased passivation of the $\mathrm{Yb}$ ions, rather than another effect related to the energy transfer from the QD.
The $\mathrm{Yb}$ luminescence decay profile is well described by a biexponential fit for all excitation wavelengths that were tested. This is possibly due to a number of competing rates present that stem from the variety of local environments of the $\mathrm{Yb}$ ions (closer to the core vs closer to the surface). Due to this complexity, we used the intensity weighted average of the two lifetimes for our analysis. Since the observed lifetimes for $\mathrm{Yb}$ are much longer than the QD band edge $(6.4 \mu \mathrm{s}$ upon $760 \mathrm{~nm}$ excitation and $9.1 \mu \mathrm{s}$ under $450 \mathrm{~nm}$ excitation), as seen in Figure 4c, we can assume that the Yb NIR emission is insensitive to the upstream kinetics of the energy transfer mechanism. The mechanism could be through a surface based $\mathrm{Yb}$ induced trap, which would be consistent with previous literature accounts in both thin film InP ${ }^{23}$ as well as recent work in doped perovskite QD's. ${ }^{18}$ Alternatively, the mechanism could involve trap state that is native to the InP, which has been seen previously in the literature.$^{20}$ In either case, the cause of the trap does not affect the ability of the InP to transfer energy to $\mathrm{Yb}$, and since the mechanism is very difficult to determine we cannot determine exactly which option it is. The mechanism of energy transfer is poorly understood, and beyond the scope of this work. In our system, the $\mathrm{Yb}$ is further from the lattice, likely reducing the wave function overlap between the band edge and the Yb-related trap state, which would slow down the 
energy transfer dramatically. This is likely why we observe any remaining band-edge photoluminescence after shell overgrowth, and why the PLQY of exciting the InP and emitting from the $\mathrm{Yb}$ remains low. The coupling may be improved in the future through materials selection and nanostructure tuning.

The intrinsic lifetime of the $\mathrm{Yb}^{3+}$ in our system is much longer ( $495 \mu$ s when directly excited at $974 \mathrm{~nm}$ ), which is consistent with previous work on passivated lanthanide systems ${ }^{24}$, but it is not at the level of a fully passivated $\mathrm{Yb}$ ion (1.9 $\mathrm{ms}$ in $\left.\mathrm{YF}_{3}\right)^{25}$. We assume, this is due to the plate morphology of the core/shell/shell nanocrystals, where the passivation is not as complete on the short axis of the material, which would allow for increased surface quenching. Additionally, there could be some diffusion of the $\mathrm{Yb}$ ions into the shell of the material ${ }^{26}$, which would also cause the ions to be closer to the quenching surface of the nanoparticle.

The optical properties of the nanoparticles were investigated under varying $\mathrm{Yb}$ concentrations. Figure $4 \mathrm{e}$ shows the effect of differing amounts of $\mathrm{Yb}(\mathrm{TFA})_{3}$ that was added to the core InP QD's. The results can be interpreted in three general regimes, namely low, medium, and high concentration. At low concentrations there is not enough $\mathrm{Yb}$ present to have a significant interaction with the $\mathrm{QD}$, so less energy is funneled into the $\mathrm{Yb}$. In the medium concentration regime there is enough $\mathrm{Yb}$ to interact well with the QD's and take in some of the excitations that are occurring, but not so many that there is an increased amount of non-radiative interactions as seen in the high concentration regime. The effect of excitation wavelength and power was also studied, and no significant correlation was found (SI).

Using this information, we extended the range of the core/shell/shell geometry scheme to include $\mathrm{Nd}$. $\mathrm{Nd}$ has many more transitions than $\mathrm{Yb}$, but the main emission line is found at $1064 \mathrm{~nm}$ (SI). We followed the same synthetic method as for $\mathrm{Yb}$ using Nd instead, and we found that there was an appreciable NIR luminescence at the characteristic Nd line of $1064 \mathrm{~nm}$. The luminescence was very weak, probably due to competing nonradiative processes introduced by the greater density of transitions in $\mathrm{Nd}$, but the results we saw indicate that this method can be extended to other Ln ions.

\section{Conclusions}

In conclusion, we have demonstrated the synthesis of core/shell/shell nanocrystals of InP/ $\mathrm{Y}_{1-x} \mathrm{Ln}_{x} \mathrm{~F}_{3} / \mathrm{ShF}_{3}$, where $\mathrm{Ln}$ is $\mathrm{Yb}$ or $\mathrm{Nd}$ and $\mathrm{Sh}$ is Lu or Y. These particles were characterized by electron microscopy and x-ray techniques, all of which were found to be in good agreement on the morphology of the particles. Optically, these particles were found to pass excitation from the InP band edge through a trap state to the $\mathrm{Yb}^{3+}{ }^{2} \mathrm{~F}_{5 / 2}$ transition, which led to an emission in the NIR at $976 \mathrm{~nm}$ with a PLQY of $0.1 \%$. With further improvements to the efficiency of this system, it could provide a tunable and broadly absorbing, sharply emitting luminophore that would be desirable in many applications. This work provides also an insight into the mechanism of energy transfer from an QD to a Ln ion, which can help inform the design of visible to NIR downshifters.
Chemicals: QD Synthesis: Indium (III) acetate (99.99\%, Sigma-Aldrich), myristic acid (Sigma Grade, 99\%, Sigma-Aldrich), 1-octadecene (ODE) (Technical Grade, 90\%, SigmaAldrich), tris(trimethylsilylphosphine) (TMSP) (98\%, STREM Chemicals), trioctyl phosphine (TOP) (97\%, STREM Chemicals). Solvents and Antisolvents: Acetone, hexanes (99\%, Anhydrous, Sigma-Aldrich), toluene (99.8\%, Anhydrous, Sigma-Aldrich), ethanol (Absolute, Sigma-Aldrich), chloroform (HPLC Grade, Fischer), ethyl acetate (99.5\%, Sigma-Aldrich), isopropyl alcohol (99.5\%, Sigma-Aldrich). Treatment Materials: Tetrabutylammonium fluoride trihydrate (TBAF) (97\%, Sigma-Aldrich), hydrofluoric acid (HF) (48 wt\% in water, 99.99\%, Sigma-Aldrich). Rare Earth Precursors: Yttrium (III) oxide (99.99\%, GFS Chemicals), ytterbium (III) oxide (99.9\%, GFS Chemicals), neodymium (III) oxide (99.9\%, GFS Chemicals), lutetium (III) oxide $(99.9 \%$, Alfa Aeser), trifluoroacetic acid (TFA) (VWR Biotech grade, VWR), oleylamine (OLA) (98\% primary amine, Sigma-Aldrich), oleic acid (OA) (Technical Grade, 90\%, Sigma-Aldrich). Schlenk Line Inert Gas: Argon (99.9995\%, Praxair with additional Nupure purifier as specified below)

\section{Preparation of InP Quantum Dots}

In(Ac) 3 (1.2 mmol, $350 \mathrm{mg}$ ), myristic acid (3.6 mmol, 822 $\mathrm{mg})$, and ODE (10 mL) were charged into a 3-neck flask and dried/degassed under vacuum at $110{ }^{\circ} \mathrm{C}$ for at least 1 hour, then the solution was heated to $130^{\circ} \mathrm{C}$ under ultra-pure argon (see discussion below for exact specifications). In an argon glovebox, TMSP (0.6 mmol, $152 \mathrm{mg}$ ) was dissolved in TOP (1.66 g) and shook to combine. The resultant solution was drawn into a Leur lock syringe with a 21 gauge needle and injected into the In(Myristate) 3 solution at $130{ }^{\circ} \mathrm{C}$ under vigorous stirring. The solution was allowed to react at 130 ${ }^{\circ} \mathrm{C}$ for 2 minutes before the reaction was ramped to $230{ }^{\circ} \mathrm{C}$. The reaction was held at $230^{\circ} \mathrm{C}$ for 15 minutes before being cooled to room temperature by removal of the heating mantle and blowing forced air over the outside of the flask.

\section{Cleaning InP Quantum Dots}

The reaction mixture of InP QDs was transferred via cannula to a dry schlenk flask that was brought into an argon glovebox. Inside, the solution was transferred to a centrifuge tube, and precipitated with excess acetone $(\sim 35 \mathrm{~mL})$. The cloudy solution was centrifuged at $8228 \mathrm{~g}$ for 6 minutes, returned to the glovebox where the clear supernatant was removed, and the resultant pellet was redispersed in $\sim 5 \mathrm{~mL}$ hexanes. The acetone precipitation-centrifugation-redispersion was repeated, and the resultant solution was stored in the glovebox for future shelling.

\section{Surface Treatment of InP QDs}

Cleaned InP QDs were treated with TBAF to passivate the surface and provide an adhesion layer for the rare earth fluorides. To do this, $100 \mathrm{nmol}$ of cleaned InP QDs in hexanes were taken and added to $0.25 \mathrm{~mL}$ of acetone that had 32.5 mg TBAF and $5 \mu \mathrm{L} \mathrm{HF}$ already mixed into it. This mixture was stirred vigorously for $\sim 2$ hours under bright, visible light illumination, after which it separated into two phases. The bottom layer was dark red and viscous while the upper layer was a lighter yellow. The upper layer was removed and discarded, and the lower viscous layer was used immediately for shellings.

Preparation of Stock Solutions for Shelling 
The rare earth shelling precursor solutions were made in a multistep procedure. First, $5 \mathrm{mmol}$ of the desired rare earth oxide $\left(\mathrm{Y}_{2} \mathrm{O}_{3}, \mathrm{Yb}_{2} \mathrm{O}_{3}, \mathrm{Nd}_{2} \mathrm{O}_{3}\right.$, or $\left.\mathrm{Lu}_{2} \mathrm{O}_{3}\right)$ was charged into a $100 \mathrm{~mL} 3$-neck flask. Then, $\sim 50 \mathrm{~mL}$ of distilled water was added, followed by $\sim 15 \mathrm{~mL}$ of pure TFA. The mixture was heated in an oil bath to $90^{\circ} \mathrm{C}$ until all of the rare earth oxide was dissolved, with a condenser column to prevent the water from evaporating. After the oxide dissolved, the water and excess TFA were removed via evaporation by removing the condenser column and septa from the flask and lowering the temperature of the oil bath to $65^{\circ} \mathrm{C}$. This resulted in $10 \mathrm{mmol}$ of rare earth trifluoroacte, which was typically a white powder. Next, $10 \mathrm{~mL}$ OA, $2.5 \mathrm{~mL}$ OLA, and $10 \mathrm{~mL}$ ODE were added to the powder, and the mixture was heated under vacuum to $120^{\circ} \mathrm{C}$. Care should be taken in this step, as there is still a lot of water bound to the TFA salts. The solution was kept under vacuum for $>2$ hours, and in the end afforded a viscous clear yellow solution. The solution was cannulated into a Schlenk flask and stored in an argon glovebox.

\section{Shelling with $\mathrm{Y}_{1-\mathrm{x}} \operatorname{Ln}_{x} \mathrm{~F}_{3}(\mathrm{Ln}=\mathrm{Yb}, \mathrm{Nd})$}

The example of $\mathrm{Y}_{0.8} \mathrm{Yb}_{0.2}$ is given, and the ratio of TFA precursors can be changed to the desired proportion. OLA (1 $\mathrm{mL})$, ODE $(7 \mathrm{~mL})$, and $100 \mathrm{nmol}$ treated InP QDs were charged into a 3 -neck flask and degassed at $100{ }^{\circ} \mathrm{C}$ until the baseline pressure of the Schlenk line was reached. The treated QDs do not disperse well into ODE, so the OLA is needed to create a homogeneous solution. While the solution is degassing, the shelling solution of $73 \mu \mathrm{L} \mathrm{Yb(TFA)})_{3}$, $223 \mu \mathrm{L} \mathrm{Y(TFA)})_{3}$, and $1704 \mu \mathrm{L}$ ODE was mixed in an argon glovebox. The TFA precursor solutions are very viscous and need to be mixed well with the ODE to create a homogeneous solution. The shelling solution was brought into a Leur lock syringe and removed from the glovebox. After degassing, the solution is ramped to $300{ }^{\circ} \mathrm{C}$ under ultra-pure argon, and when the reaction reaches temperature the shelling solution was injected at the rate of $2 \mathrm{~mL} / \mathrm{hr}$ by using a syringe pump. After the hour it took for the solution to be injected, the reaction was allowed to sit at temperature for an additional 5 minutes to allow for total reaction of the TFA precursors and was then cooled to room temperature by blowing air over the flask after the heating mantle was removed.

\section{Cleaning the Core/Shell Nanocrystals}

After the reaction mixture is cooled to room temperature, it is placed into a centrifuge tube and $\sim 35 \mathrm{~mL}$ of a $50 / 50$ mixture of acetone and ethyl acetate (the ethyl acetate is present to prevent the solution from becoming biphasic). The resultant cloudy liquid is centrifuged at $8228 \mathrm{~g}$ for 6 minutes, after which time a pellet forms at the bottom of the tube and the clear supernatant is removed and discarded. The pellet is redispersed in hexanes and precipitated again with $\sim 20 \mathrm{~mL}$ of acetone. After another round of centrifugation at $8228 \mathrm{~g}$ for 6 minutes, the supernatant is again removed and the pellet redispersed in hexanes. The final mixture ranges in color from a light pink to a deep red depending on concentration. The solution was left overnight for any insoluble impurities to precipitate out of and be removed from the solution.

Final Shelling with $\mathrm{LnF}_{3}$, where $\mathrm{Ln}=\mathrm{Y}, \mathrm{Lu}$
ODE (8 mL) and all core/shell nanoparticles from the previous shelling were mixed in a 3-neck flask and degassed at $100{ }^{\circ} \mathrm{C}$ for 1 hour. In a glovebox, the desired rare earth TFA $\left(\mathrm{Y}(\mathrm{TFA})_{3} \text { or Lu(TFA) }\right)_{3}$ ) solution $(2.25 \mathrm{~mL})$ and ODE (1.75 $\mathrm{mL}$ ) were mixed well. Again, care must be taken to thoroughly mix the viscous TFA solution with the ODE. The mixture was drawn into a Leur lock syringe and removed from the glovebox. The nanocrystal solution is heated to $300{ }^{\circ} \mathrm{C}$, and when it reaches temperature the glovebox TFA solution is injected at a rate of $2 \mathrm{~mL} / \mathrm{hr}$ by use of a syringe pump. After the injection is finished, the solution was allowed to react at temperature for another 5 minutes before the heating mantle was removed and air was blown over the flask to aid in cooling. The final solutions were typically light yellow in color. It should be noted that increasing the amount of OLA in the reaction caused less nucleation of pure $\mathrm{LnF}_{3}$ nanocrystals.

\section{Cleaning of Core/Shell/Shell Nanocrystals}

The reaction mixture was taken and the nanocrystals precipitated in a centrifuge tube with $\sim 35 \mathrm{~mL}$ of acetone. The cloudy white solution was centrifuged at $8228 \mathrm{~g}$, affording an off-white pellet and a clear supernatant. The supernatant was discarded, and the precipitation-centrifugation-redispersal repeated with acetone-hexanes-minimal IPAhexanes. The final IPA wash was necessary to remove some impurities that remained due to the solution becoming biphasic. The final solution typically appeared to be a clear light yellow or red.

\section{Size Selective Precipitation}

The cleaned core/shell/shell nanoparticles were size selectively precipitated to remove impurities and increase sample homogeneity. This was done by taking out the required amount of the final solution from the previous step into a centrifuge vial and adding acetone dropwise until the solution became cloudy. The cloudy mixture was swirled around, and the cloudiness disappeared. This process was repeated until swirling did not cause the solution to become clear, at which point the mixture was centrifuged at $8228 \mathrm{~g}$ for 5 minutes. The pellet was collected by decanting the supernatant, redispersed in hexanes, and the process was repeated once.

\section{ASSOCIATED CONTENT}

Supporting Information. Experimental details on the material and optical characterizations, supplementary results and discussions.

\section{AUTHOR INFORMATION}

\section{Corresponding Author}

*E-mail: paul.alivisatos@berkeley.edu

Funding Sources

Notes

The authors declare no competing financial interests.

\section{ACKNOWLEDGMENT}

The authors thank Brent Koscher for general advice and Matthew Marcus and Sirine Fakra of ALS beamline 10.3.2 for help 
with EXAFS measurements. The work was supported by the Light-Material Interactions in Energy Conversion, an Energy Frontier Research Center funded by the U.S. Department of Energy, Office of Science, Office of Basic Energy Sciences, under Contract DE-AC02-05CH11231, part of the EFRC at Caltech under DE-SC0001293. Work at the Molecular Foundry was supported by the Office of Science, Office of Basic Energy Sciences, of the U.S. Department of Energy under Contract No. DE-AC02-05CH11231. This research used resources of the Advanced Light Source, which is a DOE Office of Science User Facility under contract no. DE-AC02-05CH11231.

\section{REFERENCES}

(1) Goldschmidt, J. C.; Fischer, S. Upconversion for Photovoltaics - a Review of Materials, Devices and Concepts for Performance Enhancement. Adv. Opt. Mater. 2015, 3 (4), 510535.

(2) Huang, X.; Han, S.; Huang, W.; Liu, X. Enhancing Solar Cell Efficiency: The Search for Luminescent Materials as Spectral Converters. Chem. Soc. Rev. 2013, 42 (1), 173-201.

(3) Correia, S. F. H.; de Zea Bermudez, V.; Ribeiro, S. J. L.; André, P. S.; Ferreira, R. A. S.; Carlos, L. D. Luminescent Solar Concentrators: Challenges for Lanthanide-Based Organicinorganic Hybrid Materials. J. Mater. Chem. A 2014, 2 (16), 5580-5596.

(4) Wang, F.; Banerjee, D.; Liu, Y.; Chen, X.; Liu, X. Upconversion Nanoparticles in Biological Labeling, Imaging, and Therapy. Analyst 2010, 135 (8), 1839.

(5) Vetrone, F.; Naccache, R.; Zamarrón, A.; Juarranz de la Fuente, A.; Sanz-Rodríguez, F.; Martinez Maestro, L.; Martín Rodriguez, E.; Jaque, D.; García Solé, J.; Capobianco, J. a. Temperature Sensing Using Fluorescent Nanothermometers. ACS Nano 2010, 4 (6), 3254-3258.

(6) Bettinelli, M.; Carlos, L.; Liu, X. Lanthanide-Doped Upconversion Nanoparticles. Phys. Today 2015, 68 (9), 3844.

(7) Auzel, F. Upconversion and Anti-Stokes Processes with F and D Ions in Solids. Chem. Rev. 2004, 104 (1), 139-173.

(8) Wang, X.; Valiev, R. R.; Ohulchanskyy, T. Y.; Ågren, H.; Yang, C.; Chen, G. Dye-Sensitized Lanthanide-Doped Upconversion Nanoparticles. Chem. Soc. Rev. 2017, 46 (14), 4150-4167.

(9) Moore, E. G.; Samuel, A. P. S.; Raymond, K. N. From Antenna to Assay: Lessons Learned in Lanthanide Luminescence. Acc. Chem. Res. 2009, 42 (4), 542-552.

(10) Hu, J.-Y.; Ning, Y.; Meng, Y.-S.; Zhang, J.; Wu, Z.-Y.; Gao, S.; Zhang, J.-L. Highly near-IR Emissive Ytterbium ${ }^{3+}$ Complexes with Unprecedented Quantum Yields. Chem. Sci. 2017, 8 (4), 2702-2709.

(11) Beeby, A.; Clarkson, I. M.; Dickins, R. S.; Faulkner, S.; Parker D.; Royle, L.; de Sousa, A. S.; Williams, J. A. G.; Woods, M. NonRadiative Deactivation of the Excited States of Europium Terbium and Ytterbium Complexes by Proximate EnergyMatched $\mathrm{OH}, \mathrm{NH}$ and $\mathrm{CH}$ Oscillators: An Improved Luminescence Method for Establishing Solution Hydration States. J. Chem. Soc. Perkin Trans. 2 1999, 2 (3), 493-504.
(12) Chen, D.; Wang, Y.; Yu, Y.; Huang, P.; Weng, F. Quantum Cutting Downconversion by Cooperative Energy Transfer from Ce3+ to Yb3+ in Borate Glasses. J. Appl. Phys. 2008, 104 (11), 1-4.

(13) Stephan, M.; Zachau, M.; Gröting, M.; Karplak, O.; Eyert, V.; Mishra, K. C.; Schmidt, P. C. A Theoretical Investigation of $4 \mathrm{f} \rightarrow 5 \mathrm{~d}$ Transition of Trivalent Rare Earth Ions in Fluorides and Complex Oxides. J. Lumin. 2005, 114 (3-4), 255-266.

(14) Alivisatos, A. P. Semiconductor Clusters, Nanocrystals, and Quantum Dots. Science 1996, 271 (5251), 933-937.

(15) Reiss, P.; Protière, M.; Li, L. Core/shell Semiconductor Nanocrystals. Small 2009, 5 (2), 154-168.

(16) Martín-Rodríguez, R.; Geitenbeek, R.; Meijerink, A Incorporation and Luminescence of $\mathrm{Yb3}+$ in $\mathrm{CdSe}$ Nanocrystals. J. Am. Chem. Soc. 2013, 135, 13668-13671.

(17) Creutz, S. E.; Fainblat, R.; Kim, Y.; De Siena, M. C.; Gamelin, D. R. A Selective Cation Exchange Strategy for the Synthesis of Colloidal Yb3+-Doped Chalcogenide Nanocrystals with Strong Broadband Visible Absorption and Long-Lived NearInfrared Emission. J. Am. Chem. Soc. 2017, 139 (34), 1181411824.

(18) Milstein, T. J.; Kroupa, D. M.; Gamelin, D. R. Picosecond Quantum Cutting Generates Photoluminescence Quantum Yields Over 100\% in Ytterbium-Doped CsPbCl 3 Nanocrystals. Nano Lett. 2018, acs.nanolett.8b01066.

(19) Battaglia, D.; Peng, X. Formation of High Quality InP and InAs Nanocrystals in a Noncoordinating Solvent. Nano Lett. 2002, 2,1027-1030.

(20) Mićić, O. I.; Sprague, J.; Lu, Z.; Nozik, A. J. Highly Efficient BandEdge Emission from InP Quantum Dots. Appl. Phys. Lett. 1996, 68, 3150-3152.

(21) Talapin, D. V.; Gaponik, N.; Borchert, H.; Rogach, A. L.; Haase, M.; Weller, H. Etching of Colloidal InP Nanocrystals with Fluorides: Photochemical Nature of the Process Resulting in High Photoluminescence Efficiency.J. Phys. Chem. B 2002, 106 (49), 12659-12663.

(22) Ye, X.; Chen, J.; Engel, M.; Millan, J. A.; Li, W.; Qi, L.; Xing, G.; Collins, J. E.; Kagan, C. R.; Murray, C. B. Competition of Shape and Interaction Patchiness for Self-Assembling Nanoplates. Nat. Chem. 2013, 5 (6), 466-473.

(23) Taguchi, A.; Takahei, K.; Horikoshi, Y. Multiphonon-Assisted Energy Transfer between Yb 4f Shell and InP Host. J. Appl. Phys. 1994, 76 (11), 7288.

(24) Fischer, S.; Bronstein, N. D.; Swabeck, J. K.; Chan, E. M.; Alivisatos, A. P. Precise Tuning of Surface Quenching for Luminescence Enhancement in Core-Shell Lanthanide-Doped Nanocrystals. Nano Lett. 2016, 16 (11), 7241-7247.

(25) Watts, R. K.; Richter, H. J. Diffusion and Transfer of Optical Excitation in YF3: Yb, Ho. Phys. Rev. B 1972, 6 (4), 1584-1589.

(26) Hudry, D.; Busko, D.; Popescu, R.; Gerthsen, D.; Abeykoon, A. M. M.; Kübel, C.; Bergfeldt, T.; Richards, B. S. Direct Evidence of Significant Cation Intermixing in Upconverting Core@Shell Nanocrystals: Toward a New Crystallochemical Model. Chem. Mater. 2017, 29 (21), 9238-9246. 
Insert Table of Contents artwork here

1

2

3

4

5

6

7

8

9

10

11

12

13

14

15

16

17

18

19

20

21

22

23

24

25

26

27

28

29

30

31

32

33

34

35

36

37

38

39

40

41

42

43

44

45

46

47

48

49

50

51

52

53

54

55

56

57

58

59

60
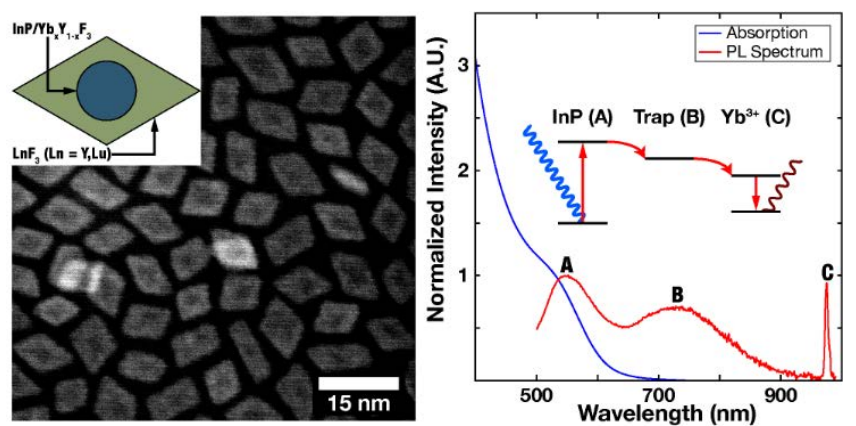

ACS Paragon Plus Environment 